\title{
PANORÁMICA DE LA ENSEÑANZA DE LA IGLESIA SOBRE LAS VOCACIONES
}

DOI: https://doi.org/10.52039/seminarios.v62i218.107

VINCENT NICHOLS*

Son de todos conocidas las tres pinturas de Caravaggio que se guardan en la iglesia romana de San Luis de los Franceses y que cuentan la historia de san Mateo. La primera, titulada "La vocación de san Mateo», constituye un excelente punto de partida para las presentes reflexiones en torno a la doctrina vocacional de la Iglesia.

Como ocurre con todas las pinturas de Caravaggio, la luz desempeña un papel muy importante en su composición. Así, en «La vocación de san Mateo» vemos la luz de la eternidad que surge desde detrás de la figura de Jesús y fluye hacia la escena del cobrador de impuestos, Mateo, que está sentado a su mesa. Jesús lo convoca a través de una llamada que está rodeada de luz celestial porque proviene del Padre. La mano de Jesús que llama es inconfundiblemente la misma mano de Adán en la obra maestra de Miguel Ángel en la Capilla Sixtina. Jesús, el segundo Adán, Ilama a Mateo a una fidelidad que sólo él puede dar.

Esto hace evidente el primer aspecto de la doctrina de la Iglesia sobre la vocación: Dios es la fuente de toda vocación.

Al observar la pintura, reparamos en que Pedro está acompañando a Jesús. La llamada a Mateo tiene como finalidad convertirse en uno de los compañeros de Jesús. La llamada y su cumplimiento tienen lugar en el contexto de la Iglesia.

En tercer lugar, Mateo es llamado en medio de la realidad de su vida cotidiana y de su trabajo. De hecho, el grupo que está reunido en torno a la mesa de los impuestos aparece vestido al estilo del siglo XVI, mientras que Jesús y Pedro llevan vestiduras propias de la iconografía del Nuevo Testamento. Todo ello nos indica que la llamada de Dios se dirige a nosotros contando con lo que somos, con nuestros defectos y en medio de nuestras realidades cotidianas.

Estos tres aspectos aportan la estructura de la siguiente reflexión, a la que se unen por último las implicaciones prácticas de esta doctrina.

* Vincent Nichols es cardenal y arzobispo de Westminster (Inglaterra). 


\section{ORIGEN DIVINO DE CADA VOCACIÓN}

a) La primera expresión de la misericordia de Dios es el regalo de la vida con una finalidad. Cada vida tiene un propósito dado por Dios. Esto es realmente una buena noticia, es el fundamento del mensaje evangélico. Mostrar esta vedad a la gente de hoy constituye una verdadera obra espiritual de misericordia.

El papa san Juan Pablo Il describía la naturaleza de este propósito común a todos los hombres cuando afirmaba que «el amor es la fundamental y originaria vocación de todo ser humano» ${ }^{1}$. Así pues, ¡qué regalo en la vida es saber que nuestro propósito básico es amar a toda costa, en lugar de tener éxito a toda costa! Tal impulso de amar suscita una inquietud en el corazón humano que encuentra su plenitud en el amor de Dios. La incesante búsqueda de Dios es una verdad absolutamente fundamental de nuestra condición humana ${ }^{2}$. Sin embargo, por respeto a su don del libre albedrío, Dios permanece escondido.

En esta línea, la exhortación apostólica Pastores dabo vobis señala que «la historia de cada vocación sacerdotal, como la de toda vocación cristiana, es la historia de un inefable diálogo entre Dios y los seres humanos, entre el amor de Dios que llama y la libertad de los individuos que le responden amorosamente» (PDV 36, 1).

b) «En la búsqueda de Dios, rápidamente caemos en la cuenta de que ninguno es autosuficiente. Más bien somos llamados, a la luz de la fe, a superar el egocentrismo, atraídos por la Santa Faz de Dios y por la tierra sagrada del prójimo, para una experiencia de comunión cada vez más profunda». Esta afirmación de la constitución apostólica Vultum Dei quaerere, nos recuerda que esta experiencia de comunión surge de la llamada al discipulado y que se trata de una comunión a la vez con las demás personas y con Dios en Cristo ${ }^{3}$. De ahí nuestra comprensión trinitaria de la vocación: la vocación tiene su origen en la voluntad del Padre; encuentra expresión en y a través del Verbo encarnado; su dinamismo, incluida esta comunión, es obra del Espíritu Santo.

Por otra parte, cada vocación conlleva, como veremos, el doble movimiento de la Trinidad: su comunión interna y su apertura a la misión. Esto es lo que hace de todos los bautizados discípulos y misioneros, sabiendo que el discipulado es incompleto si le falta la misión y que la misión es imposible sin discipulado. Tal raíz trinitaria de la vocación, como del discipulado y de la

1. Juan Pablo II, Familiaris consortio, 11.

2. Papa Francisco, constitución apostólica Vultum Dei quaerere (2016), 1.

3. Ibid. 
misión, muestra cómo la doctrina de la Iglesia sobre la vocación se arraiga al mismo tiempo en el misterio de Dios y del hombre. «El misterio de las vocaciones es mistagógico, y por tanto brota una y otra vez del Misterio (de Dios) para conducir de vuelta al misterio (de la humanidad), o no es nada» ${ }^{4}$.

c) La llamada misericordiosa de Dios al amor encuentra su expresión en la vocación a ser discípulos y misioneros de Cristo. Pero el discipulado debe desplegar ahora una forma específica en la vida de cada persona. En un famoso texto, san John Henry Newman resume los rasgos principales de la vocación personal:

Dios me ha creado para prestarle un determinado servicio: me ha encomendado una obra que no ha encargado a otro. Tengo una misión; quizá nunca lo sepa en esta vida, pero me lo dirán en la otra. De alguna forma soy necesario para sus fines: tan necesario en mi puesto como un arcángel en el suyo. Soy partícipe de esta gran obra; soy un eslabón de una cadena, un vínculo de conexión entre personas. Él no me ha creado para nada. Yo haré el bien, realizaré este trabajo; seré un ángel de paz, un predicador de la verdad en mi puesto ${ }^{5}$.

La Iglesia entiende los elementos de este "servicio específico» que se expresan típicamente en uno de los estados de vida recomendados por la Iglesia y en el trabajo.

En primer lugar, los estados de vida. Un cristiano expresa su discipulado viviendo como persona consagrada, ministro ordenado o laico, sea en la soltería o en el matrimonio. La creciente comprensión del matrimonio como una vocación es un rasgo llamativo del desarrollo de la doctrina de la Iglesia en los últimos cincuenta años, culminando con el título de la reciente asamblea del Sínodo de los obispos. En medio de muchos debates y controversias, quizá se ha reflexionado poco en la primera palabra del título del Sínodo: «La vocación y misión de la familia en la Iglesia y en el mundo contemporáneo». Paralelamente a esta doctrina en desarrollo sobre el matrimonio y la familia como una vocación, contemplamos un creciente número de fieles laicos, solteros y célibes, que contribuyen significativamente a la vida de nuestras Iglesias locales. El lugar de este estado de vida en la doctrina de la Iglesia exige una mayor profundización ${ }^{6}$.

El otro elemento clave en el servicio específico de una persona es su trabajo. La doctrina de la conexión entre vocación y trabajo se remonta varios siglos atrás, pero su más reciente y plena expresión es la encíclica de san Juan

4. Obra pontificia para las vocaciones eclesiásticas, Nuevas vocaciones para una nueva Europa (In Verbo tuo), 8.

5. J. H. Newman, Meditations and Devotion: «Hope in God-Creator».

6. Obra pontificia para las vocaciones eclesiásticas, Nuevas vocaciones para una nueva Europa, 13a. 
Pablo II, Laborem exercens, donde recuerda que «el trabajo constituye una de las dimensiones fundamentales de la existencia terrena de una persona y de su vocación ${ }^{7}$. El trabajo, como es definido en esta encíclica, no es sólo un empleo pagado, sino también el trabajo de sacar adelante a los miembros de una familia, el trabajo voluntario y el trabajo artístico. La Iglesia valora todo trabajo como un servicio a los demás, sea o no remunerado.

Sin embargo, el trabajo es sólo una de las dimensiones de la vocación de cada persona. La tendencia actual a identificar totalmente la vocación de una persona con su trabajo no es parte de la doctrina de la Iglesia. Históricamente, la idea de que el trabajo define la vocación tiene su origen en la enseñanza de los reformadores protestantes ${ }^{8}$.

En contraste, la doctrina católica sobre la vocación contempla a Dios que llama a cada persona al amor como un discípulo de Cristo en un particular estado de vida y trabajando al servicio de los demás. Este es su único y específico servicio.

\section{LA DIMENSIÓN COMUNITARIA DE LA VOCACIÓN}

Aunque cada servicio específico es vivido por un individuo, ninguna vocación se establece o desarrolla sin una comunidad. En medio de una cultura tan individualista como la nuestra, algunas personas pueden deslizarse inconscientemente hacia una comprensión narcisista de la vocación: esto sólo nos concierne a «Dios y a mí». Consideremos a un pianista que ofrece un concierto como solista: aparentemente está en el escenario realizando todo solo y solo él recibe los aplausos. Se comprende que pueda pensar que todo gira únicamente en torno a sí. Pero su arte es el fruto de la dedicación de sus padres y maestros, de los fabricantes de pianos, de los compositores, etc. Hasta los intérpretes más geniales tienen la humildad suficiente para reconocer esto. En contraste, algunos cristianos entusiastas llegan a pensar que su finalidad real es ambicionar no los mejores dones de los que habla san Pablo, sino el estrellato religioso. Como alguien dijo acerca de los ingleses: un inglés es un hombre que habiéndose hecho a sí mismo, alaba a su creador.

Al contrario, cada vocación tiene una madre, y la madre de nuestra vocación es la Iglesia ${ }^{9}$. Esta afirmación radical es parte de la doctrina de la Iglesia. Reflexionando sobre esto, yo sugeriría que esta condición maternal de la Iglesia con respecto a las vocaciones incluye tres cualidades esenciales: alabanza, servicio y alegría.

7. Juan Pablo II, Laborem exercens, 11 2013.

8. Cf. The Reformation and Vocation, en D. Hoyle, The Disciples' Call, 101, Bloomsbury

9. Concilio Vaticano II, Optatam totius, 2. 
La primera es la alabanza a Dios. Fomentando una orientación a la alabanza, la Iglesia crea un contexto en el cual el oído del alma se mantiene abierto a la llamada de Dios. Sin esto, no hay suelo, o humus, donde una vocación pueda ser sembrada. "La Iglesia es casa de misericordia, y su suelo es donde las vocaciones echan raíces, maduran y dan fruto» ${ }^{10}$.

Otro elemento que nutre nuestra vocación es el servicio. La vocación es una llamada al desprendimiento y encuentra su expresión en el servicio a los demás. Después se nutre en una comunidad que valora el servicio, especialmente el servicio a los necesitados.

En tercer lugar $-y$ aquí hablo de mi experiencia personal-, cuando existe alegría en la Iglesia, cuando los religiosos y los sacerdotes están llenos de gozo, entonces el terreno que recibe las semillas de la vocación es especialmente fértil.

Esta naturaleza «interactiva» de la vocación se observa en cada una de sus etapas. Por ejemplo, la familia, primer ámbito de germinación de las vocaciones, constituye en sí misma un despliegue de la vida trinitaria, que difunde la vida y el amor por medio de una continua creación. La oración de la familia, la celebración de los sacramentos de la iniciación cristiana, las acciones de servicio mutuo que caracterizan la vida familiar, el comer juntos, las conversaciones entre diversas generaciones, el papel de los abuelos, todo esto y mucho más son interacciones que constituyen el suelo fecundo de alabanza, servicio y alegría en donde el Espíritu siembra las semillas de diversas vocaciones.

Una vez que una vocación comienza a desarrollarse en la vida de un discípulo, su despliegue acontece gracias a un discernimiento que tiene lugar en un contexto amoroso. «El camino vocacional se emprende junto a los hermanos y hermanas que el Señor nos ha dado... es una con-vocación» ${ }^{11}$.

La entera comunidad de la Iglesia es invitada a «asumir la responsabilidad del cuidado y el discernimiento de las vocaciones» ${ }^{12}$. Esto significa que «la pastoral vocacional está relacionada con la formación continua de la persona» ${ }^{13}$. La declaración Nuevas vocaciones para una nueva Europa expresa bien la dimensión comunitaria cuando afirma: «El discernimiento vocacional tiene lugar en el curso de procesos comunitarios concretos: liturgia y oración, comunión eclesial, servicio de caridad, la experiencia de recibir el amor de Dios y de ofrecerlo como testimonio» ${ }^{14}$.

10. Mensaje del papa Francisco para la LIII Jornada mundial de oración por las vocaciones.

11. Ibid.

12. Ibid.

13. Obra pontificia para las vocaciones eclesiásticas, Nuevas vocaciones para una nueva Europa, 26e.

14. Ibid., 27. 
Esto significa que toda la acción pastoral tiene una dimensión vocacional. Los pastores pueden desanimarse hoy en día cuando ven muy pocos indicios de vocaciones a la vida sacerdotal y religiosa ${ }^{15}$. Pero es importante que cada parroquia y escuela, cada movimiento eclesial y cada Iglesia local mantengan los oídos abiertos a la llamada de Cristo en la vida de los miembros de su comunidad. Sin presionar a la gente, necesitamos encontrar nuevas maneras de proponerles las vocaciones. Esto puede implicar a veces sugerir a un joven que considere el sacerdocio como una posibilidad. También puede implicar recordar a una pareja que ya es tiempo de contraer matrimonio. Hace apenas un par de semanas, quiso conversar conmigo una persona que llevaba conviviendo con su pareja más de treinta años sobre la conveniencia de contraer matrimonio porque su nieta le había preguntado directamente: «¿Por qué no os habéis casado la abuela y tú?». No cabe duda que los niños tienen más valor que el que nosotros llegaremos a tener jamás.

Por supuesto que hemos de ser muy cuidadosos para no presionar a la gente en lo relativo a hacer compromisos para toda la vida. Otros testimonios y modelos de comportamiento son accesibles, y podemos utilizarlos.

Muchos de nosotros hemos seguido los juegos olímpicos de Río. Quizá hayan escuchado que a Gran Bretaña le fue bastante bien. En particular, el equipo inglés de ciclismo lo hizo muy bien. Dos de los más exitosos ciclistas fueron Jason Kenny y Laura Trott, ganando entre ambos diez medallas. Hace un mes, después de su regreso triunfal a Inglaterra, contrajeron matrimonio en su comunidad católica. Laura hizo una entusiasta declaración diciendo que el día de su boda había sido el más feliz de su vida, mucho mejor que todas las medallas de oro. Escribió: «Rodeada por mi querida familia he desposado a mi mejor amigo, y ahora le puedo llamar 'Mi esposo'». El día después de la boda, Jason subió a la red una foto de Laura, aún en la cama, con este simple comentario: «¡Buenos días, señora Kenny!». Todas las vocaciones deberían compartir este tipo de alegría con su comunidad, la alegría de compartir con los demás, la alegría de reflejar el gozo de la Iglesia, la esposa de Cristo.

\section{LA VOCACIÓN SACERDOTAL}

Anteriormente he destacado la dimensión teologal y eclesiológica de la vocación como es presentada en la doctrina de la Iglesia. En efecto, esta dimensión nos revela la verdad de que la Iglesia es el ámbito donde germinan todas las vocaciones y de que la oración y el amor deben constituir el marco de trabajo y del discernimiento vocacional. Ahora fijémonos en cómo describe la doctrina de la Iglesia el carácter específico de la vocación sacerdotal.

15. Ibid., 6. 
a) En primer lugar, la vocación sacerdotal tiene un carácter específicamente cristológico. La Pastores dabo vobis nos ofrece el punto de partida: «Cristo Pastor es el origen y el modelo del ministerio sacerdotal» (PDV 23). Esta es la primera y fundamental semejanza a Cristo que exige una auténtica vocación al sacerdocio. En tal vocación debe resultar evidente que el joven desea ser un pastor que ama, más que un jefe que controla. La guía pastoral de 2012 para el cuidado de las vocaciones al sacerdocio ministerial expresa esto con claridad en su número 6, cuando dice lo siguiente: «Si el sacerdocio ministerial no tiene su origen en el amor, cae en la representación de una función, en vez del don del servicio de un pastor que ofrece su vida por el rebaño».

Añado a esto una reflexión personal. Cuando pidieron recientemente a un experimentado director diocesano de la pastoral vocacional en Inglaterra que describiese las cualidades que él verificaba en un candidato al sacerdocio, contestó que la primera cualidad es simple: «iQue aparezca!». Con esto quería decir que el joven que desea ser sacerdote debe aparecer desde el momento en que él aceptó conocer al director del centro vocacional. Él debe aparecer en los actos en los que se le espera, porque "aparecer» es una parte importante de la vida de un párroco: Aparecer a tiempo para decir la misa, aparecer para visitar a los enfermos, aparecer en su oficina, aparecer para encontrarse con sus hermanos en el sacerdocio. No parece mucho, pero "aparecer» es la humilde clave de una buena parte de la vida sacerdotal. Esta simple visión es otra manera de expresar la invitación de Cristo a dejar todo y seguirlo con la libertad de espíritu necesaria para realizar la misión que confió a sus apóstoles ${ }^{16}$. Esta simple experiencia personal refleja la doctrina de la Iglesia sobre la vocación.

Una característica cristológica del sacerdocio en la doctrina de la Iglesia sobre la vocación sacerdotal dice que «el sacerdote, como bien atestigua la doctrina del carácter de las Órdenes sagradas, se configura con Cristo Sacerdote que le permite actuar en la persona de Cristo Cabeza y Pastor» (Guía pastoral, 6). ¿Cómo podemos entender este «configurarse con Cristo Cabeza»?

No cabe duda de que este es quizá el aspecto más difícil de la llamada al sacerdocio. En la tradición de la Iglesia, este punto de su enseñanza es la base del respeto y el liderazgo que se reconoce al sacerdote. Pero con mucha frecuencia el sacerdote puede percibir tal respeto y reverencia como una afirmación de sus opiniones, preferencias y gustos por encima de los de otra gente, especialmente de aquellos que ha sido llamado a servir. Sin embargo, la cuestión de la autoridad del presbítero, o de su «ser cabeza», consiste en

16. Concilio Vaticano II, Presbytorum ordinis, 2. 
que debe ser la de Cristo y sólo como la de Cristo. Las diferencias en una comunidad parroquial sólo pueden resolverse cuando existe una referencia a Cristo y a las actitudes que nos ha enseñado. El modelo de la autoridad de Cristo implica dos aspectos: es para poner en práctica la voluntad del Padre y para ejercer la autoridad siempre como humilde servicio. Tal es el ser cabeza de Cristo con el cual todo presbítero debe configurarse.

b) Una segunda característica teológica de la vocación sacerdotal es su carácter eclesial. Aquí cito nuevamente la Guía pastoral: «Esta primera dimensión del sacramento del Orden, su carácter cristológico, es la base de su dimensión eclesiológica» (n. 6). En la medida en que es necesario que la Iglesia misma sea llamada por Cristo resucitado, los sacerdotes han recibido, por el sacramento del Orden, la capacidad de ser efectivos instrumentos para la edificación de la Iglesia, por medio de la proclamación de la Palabra, la celebración de los sacramentos y la conducción del Pueblo de Dios ${ }^{17}$. Sin estos dones la Iglesia perdería su identidad. El sacerdocio ministerial es, de ese modo, el punto clave y vital de la existencia de la Iglesia en tanto que signo eficaz de la acción de la gracia por la que Cristo resucitado edifica a la Iglesia en el Espíritu (PDV 15). Así, el ministerio del sacerdote es siempre un ministerio eclesial. El despliegue del ministerio en el servicio de la Palabra y del sacramento, en el liderazgo de una comunidad parroquial, en la obediencia al obispo y, de acuerdo a la mente de la Iglesia, en una forma célibe del amor, que plantea preguntas significativas en la vida de un hombre. Por esta razón, la Iglesia tiene el derecho y el deber de discernir las vocaciones al sacerdocio, $\mathrm{y}$ tiene el derecho y el deber de insistir en las cualidades personales que se requieren para el ministerio (PDV 8).

Aquí podemos añadir un elemento más en relación a la dimensión eclesial de la vocación sacerdotal. El sacerdote, como siervo de la Iglesia, trata de expresar el don sacramental del sacerdocio en la realidad de una vida configurada con la misión de Cristo, que es propiamente la misión universal de la Iglesia. La Guía pastoral lo señala bien: «La disponibilidad para la misión define la verdad del sacerdote en cada una de sus actividades. Esto implica desarrollar una estructura interior y un modo de ser, más que un modo de hacer, que se distingue por la valentía para ir más allá de cualquier particularidad a fin de abrir el propio corazón a las necesidades de la nueva evangelización» (n. 10).

Este énfasis en la misión y en el «modo de ser» - una frase bien precisame mueve a añadir subrayados tomados de la experiencia de sacerdotes de mi propia región en Inglaterra.

17. Cf. ibid., 4-6. 
En el periodo de la persecución de la Iglesia católica en Inglaterra durante y después de la Reforma, los sacerdotes seculares ingleses eran llamados «misioneros». Ellos aceptaron este nombre con orgullo y desde su ordenación en seminarios en Roma, o en otros lugares, estos jóvenes se comprometieron con la misión en Inglaterra, sabiendo que cuando llegaran allá, su ministerio podría terminar en cuestión de horas, semanas o meses y que serían arrestados, torturados y, con toda probabilidad, asesinados. Ellos sabían que en aquellos años de persecución ser un sacerdote católico en Inglaterra era considerado un acto de traición. Por tanto, se trataba de un estado de vida castigado con la más cruel de las muertes. Sacerdotes de aquel tiempo fueron ejecutados no por lo que hubiesen hecho, sino simplemente por lo que eran. Su mismo «estilo de vida» los llevó a la pena de muerte. Hoy, en circunstancias diversas, esta misma libertad y fuerte sentido de identidad habilita a muchos sacerdotes para ser misioneros en la nueva evangelización incluso en países lejanos a sus hogares. ¡Por esta historia y por el presente damos gracias a Dios!

c) El tercer aspecto teológico de la vocación sacerdotal que debemos destacar consiste en su carácter trinitario: la vocación está profundamente arraigada en la Trinidad. Aquí aplicamos directamente a la vocación sacerdotal aquellos puntos señalados anteriormente sobre el sentido trinitario de la vocación. Es conveniente repetirlo: cada vocación sacerdotal es una llamada del Padre. Así, en el corazón del sacerdote está la consolación y el desafío de saber que ha sido llamado por el Padre con una única finalidad: que el Padre puede entregarle al Hijo, como un regalo del Padre, para ser para siempre compañero de Jesús en la misión confiada al Hijo por el Padre. Para mí este constituye el significado más profundo del sacerdocio: esta es la voluntad del Padre, que mis manos, mi voz, mis acciones, sean entregadas a su Hijo para que, con la fuerza del Espíritu Santo, puedan ser canales de la gracia de Dios para todos a los que sirvo. Entender la vocación sacerdotal de este modo trinitario clarifica muchas cosas, pero especialmente que la finalidad de la fe, la confianza en la gracia, implica conducir a cada persona a la misma vida de Dios, Padre, Hijo y Espíritu Santo, hasta encontrar la plena y eterna felicidad.

Como dije antes, la dimensión trinitaria de la vocación conlleva además una vida en la cual la íntima comunión y la misión se integran. Para el sacerdote esto es bien concreto en su vida diaria de oración y servicio. Él busca abiertamente, en su vida sacerdotal, ambas cosas para mantenerse cerca del Señor y para ser medio para la acción del Espíritu Santo en la administración de los sacramentos y en el servicio del amor, dando y recibiendo, en su ministerio cotidiano. Para hacer esto posible, un joven necesita ser formado en el Espíritu antes de entrar al Seminario. Presbyterum ordinis establece: 
«La pastoral de las vocaciones sacerdotales se ordena a generar hombres de comunión y misión, inspirados por el nuevo mandamiento (Jn 13, 34), la fuente de la espiritualidad de comunión ${ }^{18}$. Esto nos remite a las tareas cotidianas del sacerdote, que combinan la dependencia de esta comunión en el Señor, en su sentido personal y comunitario, con un activo compromiso con la misión, con el anuncio de la fe-de palabra y de obra-en todo lugar y tiempo. La vocación sacerdotal es, en efecto, siempre para la gloria del Padre, del Hijo y del Espíritu Santo.

Estas tres dimensiones de la vocación sacerdotal: cristológica, eclesiológica y trinitaria, constituyen el núcleo de la enseñanza de la Iglesia sobre la vocación.

\section{LA DOCTRINA DE LA IGLESIA SOBRE LA VOCACIÓN EN LA PRÁCTICA}

Los documentos de la Iglesia sobre la vocación, y especialmente sobre la vocación sacerdotal, contienen mucho más de lo que esta somera visión de su doctrina ha podido comunicar. Se han hecho muchos intentos para expresar este núcleo doctrinal en términos más accesibles y para vincular esta doctrina con la práctica pastoral. El Plan nacional para las vocaciones de Inglaterra y Gales es un ejemplo. Toma la doctrina fundamental sobre la vocación, como la he sintetizado, y genera una adhesión a la definición simple de la vocación. Con esta definición como fundamento, dibuja algunos pasos que una diócesis o una parroquia puede animarse a dar para promover la cultura de la vocación, sabiendo que tal ayuda es posible si deciden dar los pasos.

En los documentos de la Iglesia hay diversas indicaciones prácticas sobre las estructuras, expectativas, roles, etc., que necesita la promoción de las vocaciones. Pero esto no es realmente parte de la doctrina de la Iglesia, aunque ciertamente forma parte de su sabiduría.

\section{CONCLUSIÓN}

Regresemos a la capilla en San Luis de los Franceses y a las pinturas de Caravaggio. La segunda de las tres pinturas es la «Inspiración de san Mateo». Allí se ve al evangelista Mateo escribiendo el Evangelio, vestido con simplicidad y sosteniendo una pluma. Al responder a la llamada de Jesús, Mateo dejó atrás todas las ventajas y ganancias de ser un recolector de impuestos y llevó consigo, para el servicio del Señor, únicamente su mejor don: su pluma. Nosotros seguramente hacemos lo mismo: entregamos nuestras mejores habilidades al Señor, en nuestro ministerio a favor de su pueblo.

18. Ibid., 7. 
La tercera pintura es «El martirio de san Mateo». En ella, siguiendo una antigua tradición, se ve a Mateo, vestido ya con ornamentos sacerdotales y celebrando la misa, siendo asesinado delante del altar. Su sangre se derrama hacia el oscuro primer plano del cuadro que, según algunos, representa una piscina bautismal, construida a los pies de un altar, de acuerdo a las detalladas normas que san Carlos Borromeo determinó en la Milán de la juventud de Caravaggio. La sangre de Mateo se mezcla, como realmente sucedió, con la sangre de Cristo, y llega a ser la fuente de nuestro renacer en el bautismo.

Hoy es imposible ver o imaginar esta pintura sin pensar en el P. Jacques Hamel, asesinado el 26 de julio, al pie del altar, cuando acababa de celebrar la santa misa.

He aquí una imagen de la fidelidad a la vocación sacerdotal. El P. Jacques, a la edad de 85 años, continuaba sirviendo en la Iglesia mucho más allá de la edad en la que hubiese podido jubilarse. Su amor al sacerdocio y el amor con el cual fue estimado por la gente, se mostraron con mucha claridad en su funeral. Fueron estos dos amores los que lo motivaron a continuar en su ministerio, incluso hasta su muerte. Yo pienso que el testimonio cotidiano de un sacerdote se sintetiza en la modalidad de su muerte, de rodillas, delante del altar, en la misma posición que él adoptó el día de su ordenación.

Nuestras luchas son diferentes, pero nosotros también tenemos que luchar, cada día, para mantener fresca la llamada original y la inspiración que nos llevó a ponernos de rodillas en el momento de nuestra ordenación. Nosotros también queremos tener la misma entrega en el momento de nuestra muerte, porque la muerte es el punto final de nuestra peregrinación, nuestra vocación final, a la cual deseamos responder con humilde integridad y amor confiando en el Señor. Es él quien nos llama a la vida, a nuestro ministerio y, a través de la muerte, a estar en su presencia para siempre. Esta es nuestra tenaz esperanza y la alegría del Evangelio que proclamamos. 